\title{
Low-Temperature Anelastic Property of Nanocrystalline Ag Fabricated by Gas Deposition Method
}

\author{
Terigele Xi $^{a}$, Ryoma Suzuki ${ }^{a}$, Hisanori Tanimoto ${ }^{a} *$ (D) \\ anstitutes of Materials Science and Applied Physics, University of Tsukuba 1-1-1 Tennodai, 305-8573, \\ Tsukuba, Ibaraki Japan.
}

Received: September 29, 2017; Revised: March 07, 2018; Accepted: April 20, 2018

\begin{abstract}
It was reported that the internal friction $\left(Q^{-l}\right)$ of nanocrystalline $(n-) \mathrm{Au}$ and $n$-Cu showed a rapid increase linearly with temperature above $\sim 200 \mathrm{~K}$. Since the rapid increase in $Q^{-1}$ decreased with the progress in the grain growth, it was suggested that the anelasticity of grain boundaries in $n$-Au and $n$-Cu was thermally activated above $\sim 200 \mathrm{~K}$. In order to pursue the intrinsic behavior of the grain boundaries in $n$-metals, the internal friction of $n$-Ag was measured and the result was compared with those of $n$-Au and $n-\mathrm{Cu}$. Similar to $n$-Au and $n-\mathrm{Cu}, Q^{-1}$ of $n$-Ag was also showed the increase linearly with temperature above $\sim 200 \mathrm{~K}$. However, the onset temperature for the linear increase in $Q^{-1}$ of $n-\mathrm{Ag}$ was slight lower than those of $n$ - $\mathrm{Au}$ and $n-\mathrm{Cu}$ and it can be attributed to the lower activation energy of the grain boundary self-diffusion in $\mathrm{Ag}$.
\end{abstract}

Keywords: anelasticity, nanocrystalline Ag, gas deposition method, grain boundary.

\section{Introduction}

Polycrystalline $(p-)$ materials composed of ultrafine grains with the mean size less than $\sim 100 \mathrm{~nm}$ are called nanocrystalline ( $n$-) materials. It is known that the mechanical property of conventional $p$-metals is mainly governed by the dislocation activity. Since the dislocation activity is considerably suppressed in $n$-metals due to the ultrafine grain size, the mechanical property of $n$-metals greatly depends on the behavior of the grain boundaries. As well as the much increased volume fraction of the grain boundary region, the state of the grain boundaries also plays an important role on characteristic properties of $n$-materials. Several investigations were suggested that the grain boundary state of $n$-materials was different from that of $p$-materials ${ }^{1,2}$. When the grain boundary state of $n$-metals is different from that of conventional $p$-metals, the mechanical property $n$-metals becomes considerably modified from the $p$-metal counterparts combined with the much increased factional volume. For the plasticity measurement, it was reported that the creep rate of $n$-Au showed a sudden increase with several orders in magnitude at the applied stress above $350 \mathrm{MPa}$ at room temperature ${ }^{3}$. At the same time, the (111) preferred orientation where the most of the crystallites aligned as the $<111>$ directions were normal to the specimen surface was changed to the random texture by the creep deformation, but the mean size was almost unchanged. Further, the independent movement of the crystallites through the grain boundary sliding with keeping the crystallite shape was observed from the scanning tunneling microscopy under the creep deformation ${ }^{3}$. These observations suggested that the crystallites are connected with the viscoelastic grain boundaries in $n$-Au. Since no grain growth was observed after the creep deformation, it was surmised that a quasitwo-phase state composed of the crystallites and the grain boundaries was attained in $n$-Au ${ }^{4}$. In contrast, the creep deformation behavior at liquid nitrogen temperature was much different from that at room temperature and the no viscoelastic nature of the grain boundaries was suggested ${ }^{3}$.

The internal friction measurement is a powerful and sensitive tool to study behavior of the grain boundaries. For the elasticity measurement, we reported that the internal friction of $n$-Au showed a rapid increase linearly with temperature above $200 \mathrm{~K}$ accompanied with a decrease in dynamic Young's modulus ${ }^{5,6}$. Since the amount became small with a progress of the grain growth by annealing, the rapid increase of internal friction above $200 \mathrm{~K}$ was attributed to a certain anelastic relaxation process in the grain boundaries. These observations indicated that in $n$-Au, the anelastic nature of grain boundaries becomes thermally activated above $\sim 200$ $\mathrm{K}$. The similar increase in the internal friction above $\sim 200 \mathrm{~K}$ was also observed for $n-\mathrm{Cu}^{7}$. Combined with the results of the creep deformation, it was suggested that the anelasticity (or viscoelasticity) of the grain boundaries thermally activated above $\sim 200 \mathrm{~K}$ in $n$-metals. In the present study, we carried out the internal friction measurement of $n-\mathrm{Ag}$ and the result was compared with those of $n-\mathrm{Au}$ and $n-\mathrm{Cu}$ in order to pursue the intrinsic behavior of grain boundaries in $n$-metals. 


\section{Experimental Methods}

High density and high purity $n$-Ag was prepared by the gas deposition method. In the gas deposition method, ultrafine metallic particles formed by the inert-gas condensation process were directly deposited on a glass substrate by using a He gas jet-flow ${ }^{5}$. The purity of He used for the gas condensation and jet-flow was kept better than $99.9999 \%$ by a purification system. In the present gas-deposition method, Ag nanoparticles were deposited on a glass substrate at $265 \mathrm{~K}$ by using a cold finger cooled by liquid nitrogen. By using the XY-stage, $n$-Ag ribbons of $2 \mathrm{~mm}$ wide, $20 \mathrm{~mm}$ long and $0.02 \mathrm{~mm}$ thick were prepared. The deposition rate, thickness increase per unit area per unit time, was about $74 \mathrm{~nm} / \mathrm{s}$. The internal friction and resonant frequency were measured by using a flexure resonant vibration $(\sim 600 \mathrm{~Hz})$ of a reed specimen, where the vibration was excited electrostatistically. The texture of $n$-Ag such as the mean grain size and preferred orientation was determined from the $\mathrm{X}$-ray diffraction measurement with $\mathrm{Cu}-\mathrm{K} \alpha$ radiation. The density of $n$-Ag was measured by Archimedes method with high purity ethanol. The mean grain size of $n$-Ag used in the present study was about $30 \mathrm{~nm}$ and the density relative to the $p$-Ag value $\left(10.50 \mathrm{~g} / \mathrm{cm}^{3}\right)$ was $97 \%$.

\section{Results and Discussion}

Figure 1 shows the temperature changes in the resonant frequency and internal friction of $n-\mathrm{Ag}$. For comparison, the data of $p$-Ag are also shown in Fig. 1. The internal friction of $n$-Ag started the gradual increase with increasing temperature at around $110 \mathrm{~K}$ and turned to the almost linear increase with the temperature above $\sim 200 \mathrm{~K}$. In contrast, the internal friction of $p$-Ag started the gradual increase at around $200 \mathrm{~K}$ and turned to the rapid increase above $300 \mathrm{~K}$. It was reported that the increase in internal friction associated with grain boundary relaxation in $p$-Ag was started at around $430 \mathrm{~K}$ for the resonant frequency of $1 \mathrm{~Hz}^{8}$.

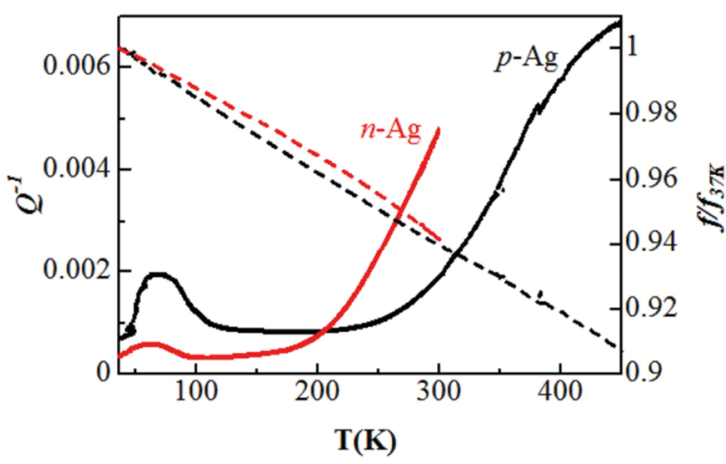

Figure 1. Temperature changes in internal friction $\left(Q^{-1}\right.$, red solid line) and resonant frequency $(f$, red dashed line) of $n-\mathrm{Ag}$. The data of internal friction (black solid line) and resonant frequency (black dashed line) of $p$-Ag are also depicted. The frequency normalized by the value at $37 \mathrm{~K}$ is shown in the figure, where $f_{37 \mathrm{~K}}$ of $n-\mathrm{Ag}$ is $\sim 300 \mathrm{~Hz}$ and that of $p$ - $\mathrm{Ag}$ is $\sim 1200 \mathrm{~Hz}$.
Figure 2 shows the changes in the X-ray diffraction pattern of $n$-Ag by annealing at elevated temperatures for $30 \mathrm{~min}$. The X-ray diffraction measurement was performed by using $\mathrm{Cu}-\mathrm{K} \alpha$ radiation for the scattering angle $(2 \theta)$ from $30^{\circ}$ to $105^{\circ}$. The increase in the mean grain size estimated from the peak broadening is depicted in Fig. 3. From Figs. 2 and 3 , the grain growth of $n$-Ag started by annealing above $400 \mathrm{~K}$ and the texture change from the strong (111) preferred orientation to the random or (100) preferred orientations by annealing above $450 \mathrm{~K}$.

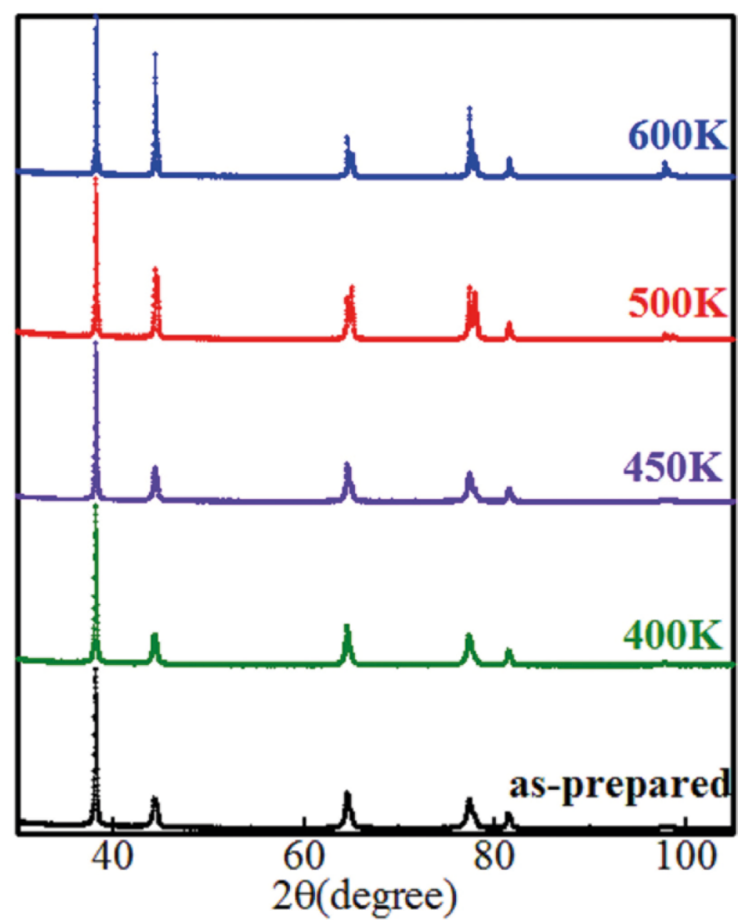

Figure 2. X-ray diffraction patterns of $n$-Ag after annealing at elevated temperatures for $30 \mathrm{~min}$.

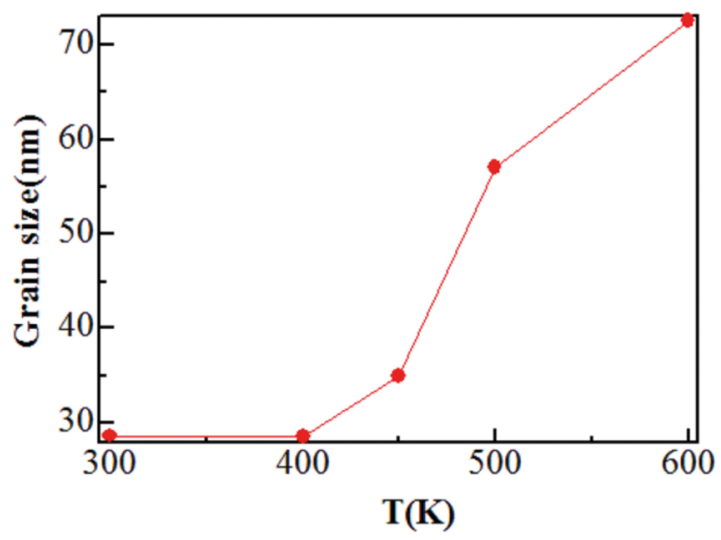

Figure 3. Change in the grain size of $n$-Ag by annealing at elevated temperatures for $30 \mathrm{~min}$. 
The changes in the resonant frequency and internal friction spectra observed for $n$-Ag by warm-up above room temperature is shown in Fig. 4. No changes in the internal friction spectrum were observed by repetition of cooldown and warm-up below $350 \mathrm{~K}$. As mentioned in Figs. 2 and 3, the apparent grain growth of $n-\mathrm{Ag}$ was observed by annealing above $400 \mathrm{~K}$. It is noted that no changes in the internal spectra were observed for $n$-Au and $n$-Cu by repetition of cool-down and warm-up below the on-set temperatures of the grain growth. When $n-\mathrm{Ag}$ was warmed up to $400 \mathrm{~K}$, a slight decrease in the internal friction and an increase in the resonant frequency were observed. When the specimen was further warmed up to $500 \mathrm{~K}$, the internal friction showed a maximum at around $450 \mathrm{~K}$ and turned to decrease rapidly at around $490 \mathrm{~K}$. Corresponding to the decrease in the linear increase of internal friction above $\sim 200 \mathrm{~K}$, the resonant frequency of $n$ - Ag showed increases after the warm-up measurements above $350 \mathrm{~K}$. The sudden and large increase in the resonant frequency in Fig. 4(b) suggests that an abnormal grain growth was occurred at around $490 \mathrm{~K}$. It is noted that dynamic Young's modulus of Ag strongly depends on the crystallographic directions; the largest is $118 \mathrm{GPa}$ along the $<111>$ direction and the lowest is $43.7 \mathrm{GPa}$ along the $<100>$ direction'. The change in the (111) preferred texture to the (100) preferred one shown in Fig. 2 indicates that the mean modulus along the reed length direction much increased due to the texture change by the abnormal grain growth. By warm-up to $670 \mathrm{~K}$, the internal friction showed a further small decrease and the resonant frequency a slight increase. These changes in the internal friction and the resonant frequency in Fig. 4 clearly suggest that the linear increase in internal friction above $\sim 200 \mathrm{~K}$ is due to a certain anelastic process at the grain boundaries.

A relaxation peak observed for $p-\mathrm{Ag}$ at around $100 \mathrm{~K}$ is known as Bordoni peak and the kink-pair formation process of dislocations was attributed ${ }^{10}$. A small peak was observed for $n-\mathrm{Ag}$ at around $80 \mathrm{~K}$. It was reported that dislocations were hardly detected in $n$ - $\mathrm{Ag}$ with grain size less than 100 $\mathrm{nm}$ prepared by inert gas condensation and compaction method $^{11,12}$. On the other hand, the observation of dislocations was reported for $n-\mathrm{Ni}^{13}$. The existence of dislocations in $n$-metal is still an open question. The peak at around $80 \mathrm{~K}$ of $n$-Ag disappeared by warm-up above $400 \mathrm{~K}$ where the grain growth was thermally activated (see Fig. 2). We tentatively surmise that the peak at around $80 \mathrm{~K}$ of $n$ - $\mathrm{Ag}$ is also owing to the grain boundaries ${ }^{3}$.

The internal friction spectra of $n-\mathrm{Ag}, n-\mathrm{Cu}$ (the mean grain size $\sim 10 \mathrm{~nm})^{7}$ and $n$-Au $(\sim 30 \mathrm{~nm})^{14}$ are compared in Fig. 5. In the figure, the internal friction due to the grain boundaries of $n-\mathrm{Cu}$ is much larger than those of $n-\mathrm{Ag}$ and $n-\mathrm{Au}$ because the mean grain size of $n$-Cu is smaller. Beyond the differences in the size, it appeared that the onset temperature of the linear increase in the internal friction for both $n$-Au and $n$-Cu was slightly higher than that for $n$-Ag. The onset

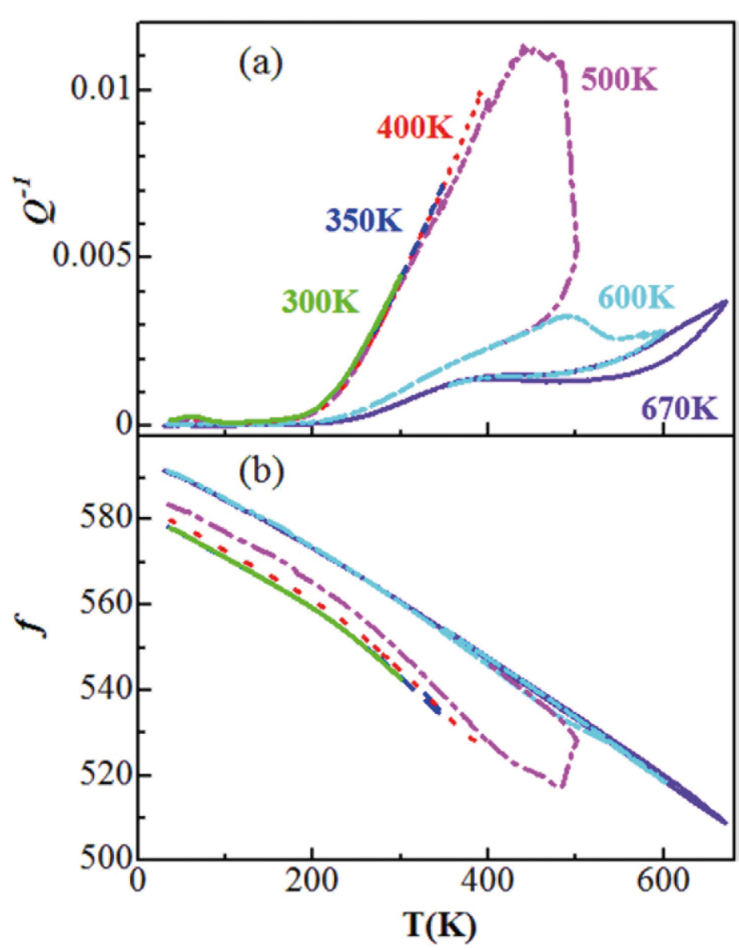

Figure 4. (a) Internal friction $\left(Q^{-1}\right)$ and (b) resonant frequency $(f)$ spectra of $n$-Ag during warm-up measurements.

temperature of the linear increase in the internal friction estimated by the linear extrapolation was $210 \mathrm{~K}$ for $n-\mathrm{Ag}$ and $230 \mathrm{~K}$ for $n-\mathrm{Au}$ and $n-\mathrm{Cu}$, respectively. The internal friction due to the grain boundaries in $n$-metals can be attributed to a certain anelastic relaxation in the grain boundaries. The probable atomistic process is reversible local site exchange of atoms ${ }^{7}$. It is expected that the local atom jumps become thermally activated at the onset temperature of the linear increase in the internal friction due to the grain boundaries. It is expected that the activation energy of the grain boundary diffusion is a rough indication for the onset temperature of the linear increase in the internal friction. Unfortunately, almost no data of the grain boundary diffusion energy of $n$-metals were reported except for the several reports ${ }^{15,16}$. In the first approximation, we refer the activation energy of the grain boundary diffusion in $p$-metals. The activation energy reported for the grain boundary self-diffusion in $p-\mathrm{Au}, p-\mathrm{Cu}$ and $p$ - $\mathrm{Ag}$ is $110.6 \mathrm{~kJ} \cdot \mathrm{mol}^{-1}, 107.2 \mathrm{~kJ} \cdot \mathrm{mol}^{-1}$ and $84.43 \mathrm{~kJ} \cdot \mathrm{mol}^{-1}$, respectively ${ }^{17}$. The activation energy of grain boundary diffusion for $p$ - $\mathrm{Ag}$ slightly lower than that of $p$-Au and $p-\mathrm{Cu}$ and it is compatible with the lower onset temperature of the linear increase in the internal friction of $n-\mathrm{Ag}$.

It is known that the grain boundary state of $n$-metals depends on the preparation method or process and the nonequilibrium grain boundaries play an important role on the properties ${ }^{18}$. Since the nanoparticles are directly deposited on the substrate by He gas jet-flow in the gas deposition method, $n$-metals with non-equilibrium grain boundaries can 


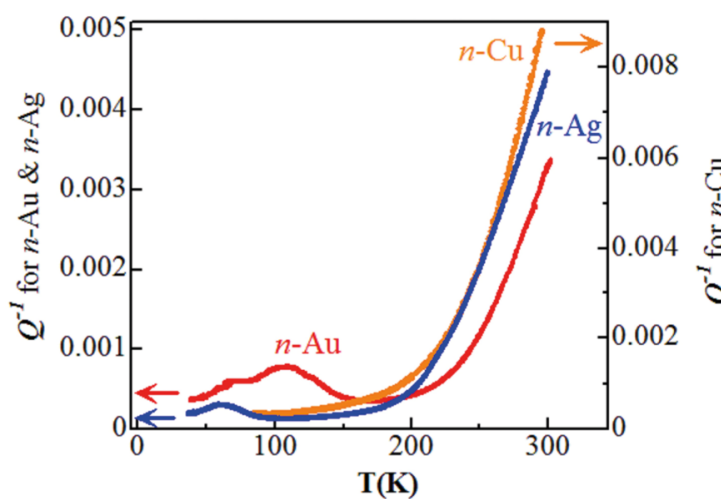

Figure 5. Comparison of the internal friction spectrum between $n$-Ag (blue), $n$-Au (red), and $n$ - $\mathrm{Cu}$ (orange).

be prepared like the severe plastic deformation methods ${ }^{19,20}$. Recently, we reported characteristic low temperature properties of $n$-Au prepared by the gas deposition method ${ }^{21}$. From the thermal analysis of $n$-Au, an endothermic tendency above $150 \mathrm{~K}$ was observed for the repetition of the cool-down and warm-up below $350 \mathrm{~K}$. The deviation to slightly lower values from the linear increase with temperature was also observed for the resistivity of $n$-Au above $\sim 150 \mathrm{~K}$. As well as the rapid increase in the internal friction, these observations suggested that the reversible state change in the grain boundaries is thermally induced at $150 \sim 200 \mathrm{~K}$. These behaviors at low temperatures indicate that a glass-transition-like change of the grain boundaries in $n$-Au is thermally activated above $150 \mathrm{~K}$ and characteristic for the non-equilibrium grain boundaries. The thermal analysis and the resistivity measurement of $n-\mathrm{Ag}$ are now in progress to survey the state change in the grain boundaries in $n$-metals as well as the atomistic process for the internal friction.

\section{Summary}

The internal friction of $n$-Au and $n-\mathrm{Cu}$ showed a rapid increase linearly with temperature above $\sim 200 \mathrm{~K}$. Since the amount decreased with a progress of the grain growth, the rapid linear increase in internal friction above $\sim 200 \mathrm{~K}$ was attributed to a certain anelastic relaxation in the grain boundaries. In the current study, the internal friction of $n-\mathrm{Ag}$ was measured and the result was compared with those of $n$-Au and $n-\mathrm{Cu}$. For $n$-Ag, the rapid increase in the internal friction was similarly observed above $\sim 200 \mathrm{~K}$, but the onset temperature of the linear increase with temperature was about $210 \mathrm{~K}$ and slightly lower than that of $n$-Au and $n-\mathrm{Cu}$. Based on the fact that the activation energy of the grain boundary self-diffusion reported for $p$ - $\mathrm{Ag}$ is lower than that of $p$ - $\mathrm{Au}$ and $p-\mathrm{Cu}$, the lower onset temperature of $n$-Ag indicates the lower activation energy for the reversible local atomic exchange motions in the grain boundaries of $n$-Ag. These observations suggest that the state change of the grain boundaries from elastic to anelastic at 150 200 K is common feature for FCC $n$-metals.

\section{Acknowledgements}

The present study was financially supported by the JSPS KAKENHI Grant 25390027 from the Japan Society for the Promotion of Science (JSPS). The authors thank Prof. Hiroshi Mizubayashi (University of Tsukuba) for valuable discussions.

\section{References}

1. Suryanarayana C. Structure and properties of nanocrystalline materials. Bulletin of Materials Science. 1994;17(4):307-346.

2. Meyers MA, Mishra A, Benson DJ. Mechanical properties of nanocrystalline materials. Progress in Materials Science. 2006;51(4):427-556.

3. Tanimoto H. Mechanical Spectroscopy of Nanocrystalline Metals and Nanometer-Thick Films: Characteristic Properties Originated in Nanostructures. Solid State Phenomena. 2012;184:42-51.

4. Yagi N, Rikukawa A, Mizubayashi H, Tanimoto H. Experimental tests of the elementary mechanism responsible for creep deformation in nanocrystalline gold. Physical Review $B$. 2006;74(14):144105.

5. Tanimoto H, Sakai S, Mizubayashi H. Anelasticity Study on Motions of Atoms in the Grain Boundary Regions in Nanocrystalline Gold. Materials Transactions. 2003;44(1):53-58.

6. Sakai S, Tanimoto H, Mizubayashi H. Mechanical behavior of high-density nanocrystalline gold prepared by gas deposition method. Acta Materialia. 1999;47(1):211-217.

7. Yagi N, Ueki A, Mizubayashi H, Tanimoto H. Anelasticity in FCC Nanocrystalline Metals. Journal of Metastable Nanocrystalline Materials. 2005;24-25:503-506.

8. Pearson S, Rotherham L. Internal Friction and Grain Boundary Viscosity of Silver and Binary Silver Solid Solutions. JOM. 1956;8(8):894-900.

9. Hellwege KH, ed. Landolt-Börnstein, Numerical Data and Functional Relationships in Science and Technology. Volume 11. Berlin, Heidelberg: Springer-Verlag; 1979.

10. Fantozzi G, Esnouf C, Benoit W, Ritchie IG. Internal friction and microdeformation due to the intrinsic properties of dislocations: The Bordoni relaxation. Progress in Materials Science. 1982;27(3-4):311-451.

11. Wang YZ, Cui P, Wu XJ, Huang JB, Cai B. Internal Friction of Nanocrystalline Silver. Physica Status Solidi (a). 2001;186(1):99104.

12. Ichikawa S, Miyazawa K, Ichinose H, Ito K. The microstructure of deformed nanocrystalline $\mathrm{Ag}$ and $\mathrm{Ag} / \mathrm{Fe}$ alloy. Nanostructured Materials. 1999;11(8):1301-1311.

13. Wu XL, Ma E. Dislocations in nanocrystalline grains. Applied Physics Letters. 2006;88(23):231911.

14. Sakai S, Tanimoto H, Kita E, Mizubayashi H. Characteristic creep behavior of nanocrystalline metals found for high-density gold. Physical Review B. 2002;66(21):214106.

15. Tanimoto H, Farber P, Würschum R, Valiev RZ, Schaefer HE. Self-diffusion in high-density nanocrystalline Fe. Nanostructured Materials. 1999;12(5-8):681-684. 
16. Nazarov AA. Grain-boundary diffusion in nanocrystals with a time-dependent diffusion coefficient. Physics of the Solid State. 2003;45(6):1166-1169.

17. Ullmaier H, ed. Landolt-Börnstein, Numerical Data and Functional Relationships in Science and Technology. Volume 25. Berlin, Heidelberg: Springer-Verlag; 1991.

18. Tucker GJ, McDowell DL. Non-equilibrium grain boundary structure and inelastic deformation using atomistic simulations. International Journal of Plasticity. 2011;27(6):841-857.
19. Valiev RZ, Alexandrov IV. Nanostructured materials from severe plastic deformation. Nanostructured Materials. 1999;12(14):35-40.

20. Sauvage X, Wilde G, Divinski SV, Horita Z, Valiev RZ. Grain boundaries in ultrafine grained materials processed by severe plastic deformation and related phenomena. Materials Science and Engineering: A. 2012;540:1-12.

21. Xi T, Sato T, Suzuki R, Tanimoto H. Glass-Transition-Like Behavior of Grain Boundaries in Nanocrystalline Gold. Materials Transactions. 2018;59(1):47-52. 